\title{
A minimal model of parallel electric field generation in a transversely inhomogeneous plasma
}

\author{
David Tsiklauri \\ Institute for Materials Research, University of Salford, Greater Manchester, M5 4WT, United \\ Kingdom.
}

\begin{abstract}
The generation of parallel electric fields by the propagation of ion cyclotron waves (with frequency $0.3 \omega_{c i}$ ) in the plasma with a transverse density inhomogeneity was studied. Using two-fluid, cold plasma linearised equations, it was shown for the first time that, in this particular context, $E_{\|}$generation can be understood by an analytic equation that couples $E_{\|}$ to the transverse electric field of the driving ion cyclotron wave. It was proven that the minimal model required to reproduce the previous kinetic simulation results of $E_{\|}$generation [Tsiklauri et al 2005, Génot et al 2004] is the two-fluid, cold plasma approximation in the linear regime. By considering the numerical solutions it was also shown that the cause of $E_{\|}$generation is the electron and ion flow separation induced by the transverse density inhomogeneity. We also investigate how $E_{\|}$generation is affected by the mass ratio and found that amplitude attained by $E_{\|}$decreases linearly as inverse of the mass ratio $m_{i} / m_{e}$. For realistic mass ratio of $m_{i} / m_{e}=$ 1836, such empirical scaling law, within a time corresponding to 3 periods of the driving ion cyclotron wave, is producing $E_{\|}=14 \mathrm{Vm}^{-1}$ for solar coronal parameters. Increase in mass ratio does not have any effect on final parallel (magnetic field aligned) speed attained by electrons. However, parallel ion velocity decreases linearly with inverse of the mass ratio $m_{i} / m_{e}$. These results can be interpreted as following: (i) ion dynamics plays no role in the $E_{\|}$generation; (ii) $E_{\|} \propto 1 / m_{i}$ scaling is caused by the fact that $\omega_{d}=0.3 \omega_{c i} \propto 1 / m_{i}$ is decreasing with the increase of ion mass, and hence the electron fluid can effectively "short-circuit" (recombine with) the slowly oscillating ions, hence producing smaller $E_{\|}$.
\end{abstract}

Keywords. waves, hydrodynamics, Sun: atmosphere, Sun: Corona, Sun: oscillations

The generation of parallel electric fields in inhomogeneous plasmas is a generic topic, which is of interest in a variety of plasma phenomena such as particle acceleration in Solar and stellar flares Fletcher(2005), auroral acceleration region and current sheets in the Earth magnetosphere (see refs. in Song \& Lysak(2006)), laboratory plasma reconnection experiments Yamada \& et al(1997) and many more. In situ and remote observations of accelerated particles often show parallel electric fields in localised double layers, charge holes or U-shaped voltage drops.

The issue of $E_{\|}$generation by the propagation of ion cyclotron waves in the plasma with a transverse density inhomogeneity is discussed in more detail in Tsiklauri(2007).

We start from two-fluid, cold (ignoring thermal pressure) plasma linearised equations Krall \& Trivelpiece(1973):

$$
\begin{gathered}
\partial_{t} \vec{V}_{e}=-\left(e / m_{e}\right)\left(\vec{E}+\vec{V}_{e} \times \vec{B}_{0} / c\right), \\
\partial_{t} \vec{V}_{i}=+\left(e / m_{i}\right)\left(\vec{E}+\vec{V}_{i} \times \vec{B}_{0} / c\right), \\
\partial_{t} \vec{B}=-c \nabla \times \vec{E},
\end{gathered}
$$




$$
\partial_{t} \vec{E}=c \nabla \times \vec{B}-4 \pi n e\left(\vec{V}_{i}-\vec{V}_{e}\right) .
$$

Hereafter subscripts under $\partial$ denote partial derivative with respect to that subscript. Uniform, background magnetic field, $B_{0}$ is in $z$-direction. Density profile is specified as a ramp, $n(x)=n_{0}\left(1+3 \exp \left[-[(x-100 \delta) /(20 \delta)]^{6}\right]\right)$ in which the central region (along $x$ direction, i.e. across $z$ ), is smoothly enhanced by a factor of 4 , and there are the strongest density gradients having a width of about $20 \delta$ around the points $x=81 \delta$ and $x=119 \delta$. Here $\delta=c / \omega_{p e}$ is the (electron) skin depth, which is a unit of grid in our numerical simulation. We use $2.5 \mathrm{D}$ description meaning that we keep all three, $x, y, z$ components of all vectors, however spatial derivatives $\partial / \partial y \equiv 0$.

In order to derive the equation that describes $E_{\|}=E_{z}$ generation, we write Eqs.(1)-(4) in $x, y, z$ component form. Omitting details of the calculation we present the final result:

$$
\left(\partial_{t t}^{2}-c^{2} \partial_{x x}^{2}+\omega_{p i}^{2}+\omega_{p e}^{2}\right) E_{\|}=-c^{2} \partial_{z x}^{2} E_{x} .
$$

Also, a similar calculation enables us to obtain the equation describing the dynamics of driving transverse electric field $E_{x}$ on an ion cyclotron wave:

$$
\begin{gathered}
\left(\partial_{t t}^{2}-c^{2} \partial_{z z}^{2}+\omega_{p i}^{2}+\omega_{p e}^{2}\right) E_{x}= \\
-c^{2} \partial_{z x}^{2} E_{\|}-\omega_{p i}^{2}\left(m_{i} / e\right) \omega_{c i} V_{i y}-\omega_{p e}^{2}\left(m_{e} / e\right) \omega_{c e} V_{e y} .
\end{gathered}
$$

Note that Eq.(6) also describes the feedback of the generated $E_{\|}$on the driving transverse electric field $E_{x}$ (see the first term on the right-hand-side). Here the notation is standard: $\omega_{p e}=\sqrt{4 \pi n_{0} e^{2} / m_{e}}$ and $\omega_{p i}=\sqrt{4 \pi n_{0} e^{2} / m_{i}}$ are electron and ion plasma frequencies; $\omega_{c(e, i)}=e B_{0} /\left(m_{(e, i)} c\right)$ are respective cyclotron frequencies.

In order to solve Eqs.(1)-(4) numerically we use the following normalisation: $t=$ $\tilde{t} \omega_{p e}^{-1}, V_{x, y, z}=\tilde{V}_{x, y, z} c, E_{x, y, z}=\tilde{E}_{x, y, z}\left(m_{e} c \omega_{p e} / e\right)=\tilde{E}_{x, y, z} E_{0}, B_{x, y, z}=\tilde{B}_{x, y, z} B_{0}$, and $(x, y, z)=c / \omega_{p e}(\tilde{x}, \tilde{y}, \tilde{z})$. In what follows we omit tilde on the dimensionless quantities. The $(x, z)$ simulation $2 \mathrm{D}$ box size is $200 \delta \times 2500 \delta$. Since we fix background plasma number density at $10^{9} \mathrm{~cm}^{-3}$ (typical value for the solar corona), $\omega_{p e}$ is then $1.784 \times 10^{9} \mathrm{rad}$ $\mathrm{s}^{-1}$ and the simulation box size is $33.6 \mathrm{~m}$ in $x$ - and $420.5 \mathrm{~m}$ in $z$-direction. $B_{0}$ was fixed at 101.5 Gauss (typical value for the solar corona), which gives $\omega_{c e} / \omega_{p e}=1 . m_{i} / m_{e}$ ratio was varied as: $45.9,91.8,183.6$ to 262.286 (realistic one is 1836). These values correspond to $1 / 40,1 / 20,1 / 10$ and $1 / 7$-th of the realistic value respectively. This yields respectively: $\omega_{c i} / \omega_{p i}=B_{0} /\left(c \sqrt{4 \pi n_{i} m_{i}}\right)=V_{A} / c=1 / \sqrt{m_{i} / m_{e}}=0.148,0.104,0.074$ and 0.062 for $x \leqslant 70$ and $x \geqslant 130$ (realistic $\omega_{c i} / \omega_{p i}=V_{A} / c$ is 0.023 ). Here parameters are similar to e.g. Tsiklauri \& et al(2005a), Tsiklauri \& et al(2005b), except for far more realistic mass ratios. Note that the simulation parameters are still somewhat artificial. Full kinetic, Particle-In-Cell (PIC) simulations employed in Tsiklauri \& et al(2005a), Tsiklauri \& et al(2005b) or in gyro-kinetic approach which uses guiding centre approximation for electrons, whilst retaining ion particle-like dynamics Génot \& et al(1999), Génot \& et al(2004) are computationally challenging. Thus, in those studies rather modest mass ratios e.g. 16 were used. Note also, that since here we do not need to resolve electron thermal motions as we are only studying electromagnetic part of the problem $\left(E_{\|}\right.$generation) our unit of spatial grid size is $\delta=c / \omega_{p e}$, the (electron) skin depth. While in full kinetic, PIC simulation Tsiklauri \& et al(2005a), Tsiklauri \& et al(2005b) the unit of grid has to be $\Delta=v_{t h, e} / \omega_{p e}$. Since in a PIC simulation typically $v_{t h, e} / c=0.1$, in the present, two-fluid approach an equivalent to PIC numerical simulation requires $(\delta / \Delta)^{2}=\left(c / v_{t h, e}\right)^{2}=10^{2}$ less grid points, thus it can be 100 times faster. For comparison a single run for mass ration 16 in Tsiklauri \& et al(2005a), Tsiklauri \& et al(2005b) takes about 8 days on parallel, 32 dual-core $2.4 \mathrm{GHz}$ Xeon processors, similar run with 


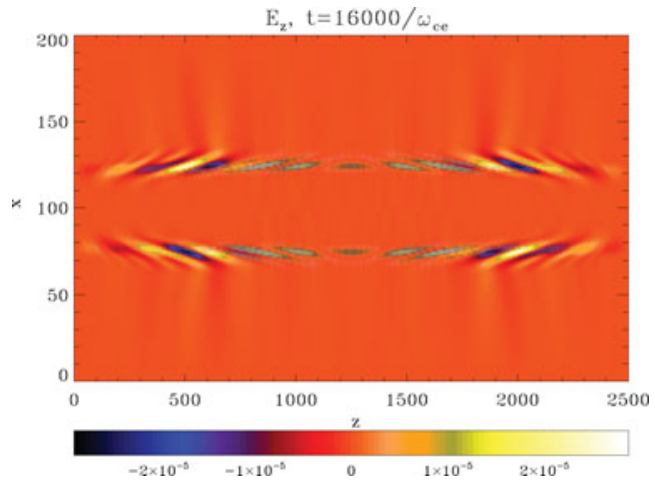

Figure 1. Contour plot of $E_{z}=E_{\|}$at time $t=16000 / \omega_{c e}$. Here $m_{i} / m_{e}=262$.

mass ratio of 262 would have taken 4 months. The numerical run presented here for the mass ratio of 262 takes 4 days with only one processor.

We solve relativistic version of Eqs.(1)-(4) numerically with a specially developed and tested FORTRAN 90 code which uses 4-th order centred spatial derivatives and 4-th order Runge-Kutta time marching. Although Alfvén speeds considered are at most $\approx 15$ $\%$ of the speed of light for $m_{i} / m_{e}=45.9$, relativistic effects were included. The simplest option becomes available in the linear regime. In Krall \& Trivelpiece(1973), appendix I, paragraph 5, it was shown that the relativistic equation of motion of a particle with charge $q$ and the rest mass $m_{0}$ can be written as

$$
\frac{d}{d t} \vec{V}=\frac{q}{\gamma m_{0}}\left[\vec{E}+\frac{\vec{V} \times \vec{B}}{c}-\frac{\vec{V}(\vec{V} \cdot \vec{E})}{c^{2}}\right],
$$

where $\gamma=\left(1-V^{2} / c^{2}\right)^{-1 / 2}$. As can be seen from the latter equation, in the linear regime, it coincides with either Eq.(1) or (2) after substituting $m_{e, i} \rightarrow \gamma_{e, i} m_{e, i}$, where $\gamma_{e, i}=\left(1-V_{e, i}^{2} / c^{2}\right)^{-1 / 2}$. Naturally, such simplified approach is only valid when there are no flows in the unperturbed state $V_{0}=0$. As can be seen below, largest attained velocities in the simulation are those of electrons, and these do not exceed $3 \%$ of speed of light. Thus, relativistic corrections play only a minor role. It should be noted, however we still retain the displacement current in Eq.(4). Note, also that the gradients in the code are resolved numerically to an appropriate precision (20 grid points across each gradient.)

Initially all perturbations are set to zero, and we start driving the $z=1$ cell with the transverse magnetic fields of the form $B_{y}=-0.05 \sin \left(\omega_{d} t\right)\left(1.0-\exp \left[-\left(t /\left(3.125 \omega_{c i}^{-1}\right)\right)^{2}\right]\right)$ and $B_{x}=-0.05 \cos \left(\omega_{d} t\right)\left(1.0-\exp \left[-\left(t /\left(3.125 \omega_{c i}^{-1}\right)\right)^{2}\right]\right)$. As in Tsiklauri \& et al $(2005 \mathrm{a})$, Tsiklauri \& et al $(2005 \mathrm{~b})$, we fixed $\omega_{d}$ at $0.3 \omega_{c i}$ (to avoid ion-cyclotron damping playing any role). $\left(1.0-\exp \left[-\left(t /\left(3.125 \omega_{c i}^{-1}\right)\right)^{2}\right]\right)$ factor ensures that these driving $B_{\perp}$ fields ramp up to their maximal values in time $t=3.125 \omega_{c i}^{-1}$. Such driving with $B_{\perp}$ of $5 \%$ of the background $B_{0}$ excites circularly polarised ion-cyclotron (IC) waves, these waves are often misquoted as Alfvén waves Génot \& et al(1999), Génot \& et al(2004), Tsiklauri \& et al(2005a), Tsiklauri \& et al(2005b). Although, in the frequency range $\omega \ll \omega_{c i}$ both left and right polarised IC waves tend to an Alfvén wave branch in the dispersion relation Krall \& Trivelpiece(1973), at frequencies $\omega \simeq 0.3 \omega_{c i}$ the correct term ion-cyclotron wave instead should be used. In the considered problem $E_{x}$ and $B_{y}$ are both components of Alfvén (ion cyclotron) wave, so these can be used interchangeably. Conventionally, Alfvénic and IC waves are more associated with magnetic field oscillation. It is in kinetic, Particle-In-Cell simulations driving by electric field is required because particles 


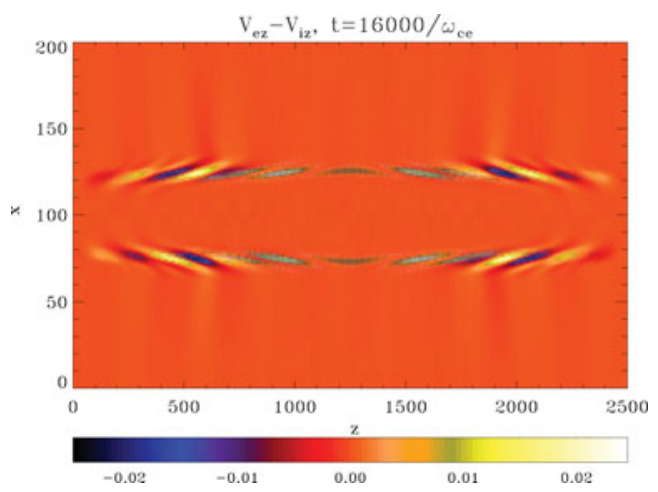

Figure 2. Contour plot of $\left(V_{e z}-V_{i z}\right) \propto j_{z}$ at time $t=16000 / \omega_{c e}$. Here $m_{i} / m_{e}=262$.

respond to electric, rather than magnetic fields. In the two-fluid simulation this is not a requirement.

The generated at the left edge $(z=1)$ IC waves propagate both in the directions of positive and negative $z$ 's. However, because of the periodic boundary conditions used (applied on all physical quantities) IC wave that travels to the direction of negative $z$ 's (to the left) re-appears on the right edge of the simulation box. The driving $B_{\perp}$ dynamics is not presented here (see for details Tsiklauri(2007)), but it resembles closely to the previous kinetic simulation results Génot \& et al(1999), Génot \& et al(2004), Tsiklauri \& et al(2005a), Tsiklauri \& et al(2005b). As in all previous phase-mixing simulations Alfvén velocity is a function of the transverse (to the background magnetic field) coordinate, $x$, i.e. $V_{A}=V_{A}(x) \propto 1 / \sqrt{n(x)}$. Thus the IC wave middle portion travels slower than the parts close to the simulation box edge. This creates progressively strong transverse gradients and hence smaller spatial scales. If resistive effects are included (these are absent here), such a configuration usually produces greatly enhanced dissipation and IC wave amplitude decays in space as $\propto \exp \left(-z^{3}\right)$ Tsiklauri \& et al(2005a), Tsiklauri \& et $\mathrm{al}(2005 \mathrm{~b})$.

The $E_{\|}=E_{z}$ field snapshot at $t=16000 / \omega_{c e}$ is shown in Fig.(1).

$E_{\|}$is generated only in the regions of density gradients i.e. around $x=81$ and $x=119$ lines. This can be explained by analysing right-hand-side (RHS) of Eq.(5). $E_{\|}=0$ at $t=0$ everywhere, however it can only be generated in the regions where $\partial_{x} E_{x} \neq 0$. The latter is true only in the density gradient regions where $E_{x}$ becomes progressively oblique propagating. Thus, Eq.(5), derived here for the first time, correctly explains the simulated process of $E_{\|}$generation by IC waves. Also, note that $E_{\|}$amplitude at time $t=16000 \omega_{c e}^{-1}$ attains value of $3 \times 10^{-5}$. This is somewhat smaller value than the one obtained in the full kinetic (PIC) simulation Tsiklauri \& et al(2005a), Tsiklauri \& et al(2005b). This is due to the different mass ratios: in the the kinetic (PIC) simulation $m_{i} / m_{e}=16$, but here it is 262. In dimensional units this $E_{\|}$corresponds to about 0.003 statvolt $\mathrm{cm}^{-1}$ or $90 \mathrm{~V} \mathrm{~m}^{-1}$, i.e. in such electric field electrons would be accelerated to the energy of $\approx 10 \mathrm{keV}$ over the distance of $100 \mathrm{~m}$. Note, however, that the generated $E_{\|}$is oscillatory in space and time. The typical values of the Dreicer electric field on the corona is few $\times 10^{-3} \mathrm{~V} \mathrm{~m}^{-1}$ Tsiklauri(2006b), which implies the obtained $E_{\|}$in our model exceeds the Dreicer value by at least four orders of magnitude, perhaps enabling the electron run away regime. This would imply that our model is more relevant to the acceleration of solar wind, rather than solving coronal heating problem. Essentially acceleration of electrons would dominate over the heating as such. However, this seems uncertain because electron and ion fluid separation cannot go on forever, and some sort of discharge should eventually 
take place. At any rate, similar kinetic simulations have shown Génot \& et al(2004) (see their Fig.(11)) that that wave energy is converted into particle energy on timescales of $10^{3} \omega_{p e}^{-1}$ (mind that the latter number is likely to be dependent on the mass ratio $m_{i} / m_{e}$ ).

In Fig. (2) we present $\left(V_{e z}-V_{i z}\right)$ which is proportional to $j_{z}$, the parallel (electron and ion flow separation) current. It can be seen from this figure that $\left(V_{e z}-V_{i z}\right)$ attains moderate values of $\approx 0.03 c$. Génot \& et al $(2004)$ stated the importance of charge separation before. However the cause of $E_{\|}$generation is actually electron and ion flow separation (see below). The latter is quite different from the electrostatic effect of charge separation, which is inherently a plasma kinetic effect. Electron and ion flow separation is a fluid-like (non-kinetic) effect, because their distribution functions remain Maxwellian at all times.

In Fig.(3) we present of the final attained parallel electric field amplitude (within 3 periods of the driving ion cyclotron wave) as a function of mass ratio, i.e. $E^{*}=$ $\max \left(\left|E_{z}\left(x, z, t_{\text {final }}\right)\right|\right)$ vs. $m_{i} / m_{e}$. We see that the amplitude attained by $E_{\|}$decreases linearly with inverse of the mass ratio $m_{i} / m_{e}$. The $x$-range in Fig.(3) is $m_{i} / m_{e}=30-$ 1836 , so that rightmost point of the dashed line enables us to grasp $E_{\|}$for the case of realistic mass ratio (i.e. 1836 ). We thus gather that $E_{\|}=0.0085 / 1836=4.630 \times 10^{-6}$ which is $4.630 \times 10^{-6} \times E_{0}=4.7 \times 10^{-4}$ statvolt $\mathrm{cm}^{-1}$ or $14 \mathrm{Vm}^{-1}$. Note that it is likely that the actual value will be even smaller if the dissipative effects (resistivity, viscosity) will be taken into account. However, these are know to be very small in the solar coronal plasma. Thus, we do not expect a big change in this result.

In Fig. (4) we show what trends in generation of parallel (magnetic field aligned) electron and ion flow as a function of the mass ratio. It follows from Fig.(4) that an increase in mass ratio does not have any effect on final parallel speed attained by electrons. However, the parallel ion velocity decreases linearly with inverse of the mass ratio $m_{i} / m_{e}$. The ratio of final attained ion and electron flow amplitudes (within 3 periods of the driving ion cyclotron wave) as a function of mass ratio, i.e. $V_{*}=$ $\max \left(\left|V_{i z}\left(x, z, t_{\text {final }}\right)\right|\right) / \max \left(\left|V_{e z}\left(x, z, t_{\text {final }}\right)\right|\right)$, shows clear scaling of $1 /\left(m_{i} / m_{e}\right)$.

The conclusions that follow from the collective analysis of Figs.(3)-(4) initially may seem counterintuitive. On one hand maximal attained $E_{\|}$amplitudes drop off as $1 / m_{i}$ (Fig.(3)). On the other hand, electron flow maximal attained amplitudes do not depend on $m_{i}$ (they all are circa $0.03 c$, see Fig.(4)), while ion flow maximal attained amplitudes are much smaller $(0.0001 c-0.00065 c)$ than that of electrons and drop off as $1 / m_{i}$ (Fig. (4)). Thus one might expect that more massive ions should produce a bigger $E_{\|}$ (since separation of electron and ion fluids is the source of $E_{\|}$and that separation is expected to be largest in the case of more massive ions, as they are slower). In fact, this is what would be expected if the polarisation drift produced by the driving IC wave is the cause of parallel electric field generation Génot \& et al(1999), Génot \& et al(2004). The latter two references use the following polarisation drift current, $j_{\perp}=\left(m_{i} n_{i} / B^{2}\right) \partial E_{\perp} / \partial t$. This equation implies that $E_{\|}$then should increase with $\propto m_{i}$. However, in Fig.(3) we see completely opposite $E_{\|} \propto 1 / m_{i}$ scaling. These results can be interpreted (reconciled) as following: (i) ion dynamics plays no role in the $E_{\|}$generation, i.e. polarisation drift has no effect in contrary to the claims of Génot \& et al(1999), Génot \& et al(2004); (ii) decrease in the generated parallel electric field amplitude with the increase of the mass ratio $m_{i} / m_{e}$ is caused by the fact that $\omega_{d}=0.3 \omega_{c i} \propto 1 / m_{i}$ is decreasing, and hence the electron fluid can effectively "short-circuit" (recombine with) the slowly oscillating ions, hence producing smaller $E_{\|}$which also scales exactly as $1 / m_{i}$.

It should be noted that since here we use two-fluid approach the generated $E_{\|}$cannot change the distribution function, which obviously remains Maxwellian, while in the previous kinetic simulation of a similar system it produced bumps in the distribution 


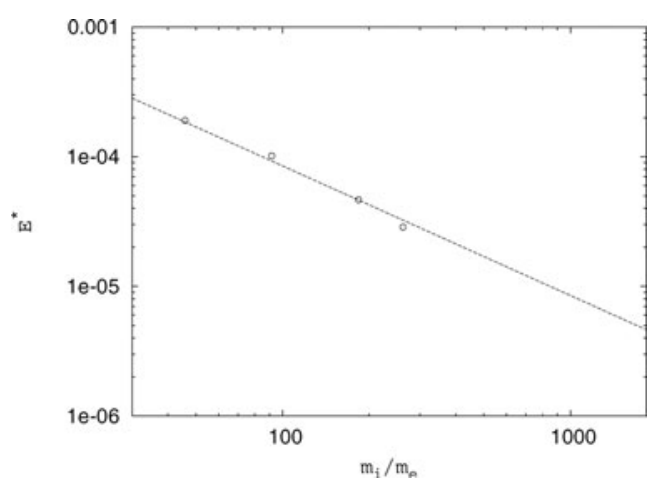

Figure 3. A log-log plot of the final attained parallel electric field amplitude (generated within 3 periods of the driving ion cyclotron wave) as a function of mass ratio. Data points correspond to $E^{*}=\max \left(\left|E_{z}\left(x, z, t_{\text {final }}\right)\right|\right)$ for $m_{i} / m_{e}=45.9,91.8,183.6$ and 262.286. The dashed line corresponds to the fit $0.0085 /\left(m_{i} / m_{e}\right)$.

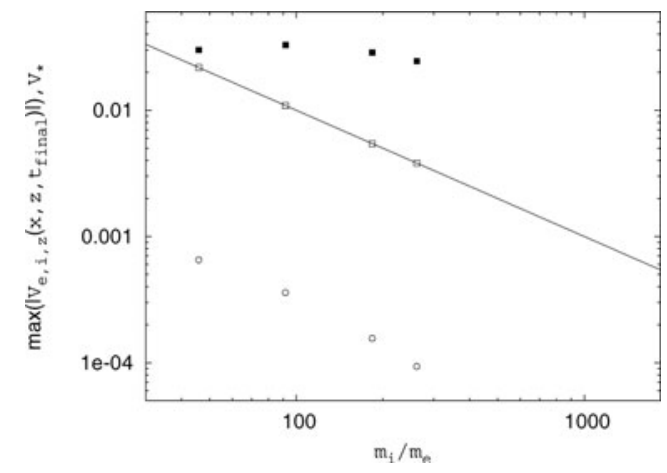

Figure 4. A log-log plot of the final attained electron and ion flow amplitudes (generated within 3 periods of the driving ion cyclotron wave) as a function of mass ratio. Solid squares correspond to $\max \left(\left|V_{e z}\left(x, z, t_{\text {final }}\right)\right|\right)$, open circles to $\max \left(\left|V_{\text {iz }}\left(x, z, t_{\text {final }}\right)\right|\right)$, while open squares show their ratio, i.e. $V_{*}=\max \left(\left|V_{i z}\left(x, z, t_{\text {final }}\right)\right|\right) / \max \left(\left|V_{e z}\left(x, z, t_{\text {final }}\right)\right|\right)$. The solid line show a fit $1 / x=1 /\left(m_{i} / m_{e}\right)$.

function as the electrons residing on the magnetic field lines with the density gradients get efficiently accelerated (see e.g. Fig.(4) in Tsiklauri \& et al(2005b)).

The generation of $E_{\|}$is a generic feature of plasmas with the transverse density inhomogeneity and in a different context this was known for decades in the laboratory plasmas Cross \& Miljak(1993), Ross \& et al(1982). Also, it should be emphasised that the two fluid description in the context of parallel electric field generation has been used before Goertz \& Boswell(1979). Moreover, Lysak(1990) has explored similar equation. It can be easily shown that our Eq.(5) can be reduced to Eq.(44) from Lysak(1990), by neglecting the displacement current and writing it in Fourier-transformed form. At first sight, this may seem to diminish the importance of our result. However, first, the inclusion of the displacement current mathematically means introduction of the time-dynamics $\left(\partial_{t t}^{2}\right.$ term in Eq.(5)) which is crucial for the correct description of $E_{\|}$generation by IC wave driving in the transversely inhomogeneous plasma; second, generally the importance of the inclusion of the displacement current in this context has been recently stressed Song \& Lysak(2006); and third, because of transverse inhomogeneity of plasma it is impossible to use Fourier-transform in the transverse spatial coordinate. It should be noted when plasma density is homogeneous no $E_{\|}$generation takes place, in our model; and 
this is corroborated both by numerical simulations (not presented here) and agrees with the Eq.(5) (when $n=$ const, the RHS of Eq.(5) is zero at all times as $E_{x}$ does not propagate obliquely). However, the relation of this claim to the observations is not entirely straightforward. Observations in the Earth's magnetosphere have for many years noted that perpendicular gradients of the perpendicular electric field are commonly seen during auroral zone crossings Mozer \& et al(1977). These are sometimes seen in the presence of perpendicular (transverse) density gradients; and the plasma density typically decreases above parallel potential drops Carlson \& et al(1998). The question here is what is generated first $E_{\|}$or $E_{\perp}$ (since obviously both are coupled via Eq.(5))? Our model also correctly reproduces the previous kinetic results Génot \& et al(1999), Génot \& et al(2004), Tsiklauri \& et al(2005a), Tsiklauri \& et al(2005b) that only electrons are accelerated (along the background magnetic field), while ions practically show no acceleration. This applies only on time scales considered i.e. $t \leqslant 16000 / \omega_{c e}$. However, in the Earth's auroral zone a significant amount of ion acceleration takes place Horwitz(1982) and Alfvénic Poynting flux is directly correlated with ion outflow in the auroral zone Strangeway \& et al(2000). Based on this one can conjecture that either processes reported by these observations acted over time scale $t \gg 16000 / \omega_{c e}$ so that ions due to their large inertia have enough time to accelerate or a mechanism other then proposed here is responsible for the ion acceleration. It should be noted that because of oscillatory nature of the obtained $E_{\|}$, it can possibly act as yet another mechanism for interpreting the peculiar hard x-ray $(>25 \mathrm{keV})$ solar flare, which is believed to be produced by a non-thermal electron beam Ofman \& Sui(2006), Nakariakov \& et al(2006).

\section{Acknowledgements}

Author is supported by the University of Salford Research Investment Fund 2005 grant and Science and Technology Facilities Council (UK) standard grant.

\section{References}

Carlson C.W., et al, 1998, Geophys. Res. Lett., 25, 2013

Cross R.C., Miljak D., 1993, Plasma Phys. Control. Fus., 35, 235

Fletcher L., 2005, Sp. Sci. Rev., 121, 141

Génot V., et al, 1999, J. Geophys. Res., 104, 22649

Génot V., et al, 2004, Ann. Geophys., 6, 2081

Goertz C.K., Boswell R.W., 1979, J. Geophys. Res., 84, 7239

Horwitz J.L., 1982, Rev. Geophys. Sp. Phys., 20, 929

Krall N.A., Trivelpiece A.W., 1973, Principles of Plasma Physics, McGraw-Hill, New York

Lysak R.L., 1990, Sp. Sci. Rev., 52, 33

Mottez F., et al, 2006, Astron. Astrophys., 449, 449

Mozer F.S., et al, 1977, Phys. Rev. Lett., 38, 292

Nakariakov V.M., et al, 2006, Astron. Astrophys., 452, 343

Ofman L., Sui L., 2006, Astrophys. J. Lett., 644, 149

Ross D.W., et al, 1982, Phys. Fluids, 25, 652

Song Y., Lysak R.L., 2006, Phys. Rev. Lett., 96, 145002

Strangeway R.J., et al, 2000, J. Geophys. Res., 105, 21129

Tsiklauri D., 2006a, New J. Phys., 8, 79

Tsiklauri D., 2006b, Astron. Astrophys., 455, 1073

Tsiklauri D., 2007, New J. Phys., 9, 262

Tsiklauri D., et al, 2005a, New J. Phys., 7, 79

Tsiklauri D., et al, 2005b, Astron. Astrophys., 435, 1105

Yamada M., et al, 1997, Phys. Plasmas, 4, 1936 